\title{
Automatic Student Attendance System using Face Recognition
}

\author{
Partha Chakraborty, Chowdhury Shahriar Muzammel, Mahmuda Khatun, Sk. Fahmida Islam, \\ Saifur Rahman
}

\begin{abstract}
The most common difficulty that every teacher faces in class room is to take the attendance of the students one by one in each and every class. For the time being many automated systems has been proposed for taking student attendance. In this paper, I introduced an automated student attendance system based on the use of unique techniques for face detection and recognition. This system automatically detects the student when he or she enters the classroom and recognizes that specific student and marks the student's attendance. This method also focuses on the specific features of different attributes such as the face, eye and nose of humans. In order to evaluate the performance of different face recognition system, different real-time situations are considered. This paper also suggests the technique for handling the technique such as spoofing and avoiding student proxy. This system helps track students compared to traditional or current systems and thereby saves time.
\end{abstract}

Keywords: Face detection, Feature Extraction, Face recognition, Eigenface, Haar Cascade Classifier, Principal Component Analysis (PCA)

\section{INTRODUCTION}

Student attendance is taken manually through the use of attendance sheets issued by department heads as part of the legislation in most learning institutions. In these sheets, students sign up for future study, which is then filled in or signed on to a computer manually. This approach is slow, time-consuming and inconsistent as some students often sign for their missing colleagues. It also makes it difficult to track the attendance of individual students in a large classroom setting. In our work, we propose the design and implementation of a face detection and recognition system to automatically detect students attending a lecture in the classroom and recognise their attendance by recognizing their faces.

While other methods of biometric authentication may be more reliable, students usually have to queue for a long time

Revised Manuscript Received on February 05, 2020.

* Correspondence Author

Partha Chakraborty*, Department of CSE, Comilla University, Comilla - 3506, Bangladesh. Email: partha.chak@cou.ac.bd

Chowdhury Shahriar Muzammel, Department of CSE, Comilla University, Comilla - 3506, Bangladesh. Email: anik.com@gmail.com Mahmuda Khatun, Department of CSE, Comilla University, Comilla 3506, Bangladesh. Email: nishucse03@gmail.com

Sk. Fahmida Islam, Department of CSE, Jahangirnagar University, Savar, Dhaka, Bangladesh. Email: jharnaju@yahoo.com

Saifur Rahman, Department of CSE, Comilla University, Comilla 3506, Bangladesh. Email: saifurrahmany43@gmail.com

(C) The Authors. Published by Blue Eyes Intelligence Engineering and Sciences Publication (BEIESP). This is an open access article under the CC BY-NC-ND license (http://creativecommons.org/licenses/by-nc-nd/4.0/) when they enter the classroom. Because of its non-intrusive nature and familiarity, face recognition is chosen because people recognize other people primarily based on their facial characteristics. The biometric (facial) system consists of an enrollment process in which the specific features of a person's face are registered in a database and then the recognition and authentication processes. The detected face (acquired from the webcam) in a picture is matched with the previously stored faces captured in this process at the time of attendance.

Many universities still use conventional methods for student attendance. Because this approach is used, many students support their peers by signing in their attendance if they are absent from the school. So while using this process, the faculty analyzes and manually manages attendance records to know the number of present and absent students. If the attendance sheet is lost, the faculty will have to attend again and in this case students who are absent will have the chance to make their present in a new sheet. The practice will also affect students as time is spent on signing, checking and sending the attendance sheet manually, in addition to being inconvenient for the lecturer. Therefore, it is necessary to develop a computerized system that can handle and allow the lecturers to easily attend and sustain the attendance. This program can be easily accessed by the faculty. The program must take careful monitoring and control of student attendance data in order to minimize the faculty's manual review of student attendance. The program analyzes all the data automatically as it is transmitted by the faculty. Instead of traditional methods, by using our face detection and recognition system, you can get a fast and effective method to collect student attendance reliably while providing safe, stable and robust system record storage where allowed.

\section{A. Contribution of Our Work}

We can get rid of common, manual and time-loss methods for marking student attendance in classrooms using this automatic method. Using face detection and recognition automated attendance can boost attendance control and management effectiveness. A software application module that faces the recognition of the images faces recorded in the video, marks the students ' register and then stores the results in a database for future study. The system uses the Eigenface recognition technique to recognize faces. The system evaluates and measures Eigenfaces which are faces made of eigenvectors. This approach also estimates the Eigenfaces to determine the presence of a person (face) and its identity. This method involves the following steps: 
Train face dataset. Next when we sense a face, we calculate Eigenface for that face. The system then compares the current face's eigenvectors and the face picture stored decides whether or not the face is detected. The final step (optional) is that the system learns to recognize faces if the unknown face is found repeatedly.

\section{RELATED WORK}

The main reason behind the system is to analyze and find solutions from other writers and recognize their suggested system imperfection and provide the best solutions. Kawaguchi developed a classroom attendance system with a new method called continual monitoring, and student attendance reported dynamically by the camera capturing the image of a student in the classroom [1]. The design of the network is simple as the class wall is fitted with two cameras. The first is a camera that captures the image of students in the classroom; the other is a sensor camera to capture the chair of a student. The program compares the image taken from a camera that records photographs and faces in the database that made a lot of time to make the attendance great. Another paper proposed by [2] implemented in the automated attendance management system a real-time computer vision algorithm. The system installed the non-intrusive camera capable of snapping images in the classroom and comparing the captured face with faces within the system from the camera's image. The approach often uses techniques that are often used in computer vision for machine learning. Haar classifiers were used in camera capture images to train. The camera's face snap converts to grayscale and retracts the files, which is then forwarded for later saving and processing on the server in 2012Used two libraries including OpenCV is a computer vision library and FLTK to implement the program. Both of these libraries contributed to the development of the interface, such as the OpenCV support algorithm [4] and FLTK. An integrated attendance management system was implemented by Kar [3] using face recognition techniques. The system provides matching requests and adding new information to the database. Request Matching's first move is to open the shutter and snap the picture after removing the front face. The next step is to classify the face with the training data and to paper the extracted face onto the main feature visualization. A smart attendance marking system that involves two differentiating algorithms such as Principal Component Analysis and Artificial Neural Network ase suggested by Jyotshana Kanti [5]. Zacharia proposed using face recognition [6] an advanced attendance management system in which only PCA was used. J.G. in their inquiry. Roshan Tharanga et al proposed an intelligent way of marking the attendance [7]. K. Senthamil Selvi et al discussed recent developments in the topic in their paper[8]. Shireesha Chintalapati et al, in their report, discussed face recognition uisng PCA, LDA, and LBPH [9].

\section{METHODLOGY}

\section{A. Principle Face Recognition (PCA) Algorithm}

In this system, we used PCA algorithm for face recognition. This is step by step algorithm overview.

Step 0: Creating and loading a training set. The training collection consists of $\mathrm{M}$ images. Where each image size is $\mathrm{N} / \mathrm{N}$

Step 1: Convert the images to face vectors in the training set. The pictures are translated to vectors of the board. Let a face image be an 8-bit intensity two-dimensional $\mathrm{N}$ by $\mathrm{N}$ array. An image can also be regarded as a squared dimension $\mathrm{N}$ vector. So a standard 50 by 50 size images becomes a 2500 dimensional space vector. A set of images, then, maps this huge space to a selection of points.

Step 2: Standardize face vectors, i.e. remove all common features. This is done by measuring and subtracting from all the facial vectors the average facial vector.

Step 3: Calculate ownership of the covariance matrix. As the main components are obtained from the covariance matrix in the PCA-based Eigenfaces method, we now calculate the own vectors from the covariance matrix. It would take a lot of time to find $K$ own vectors from $M=2500$ own vectors with $\mathrm{K}<\mathrm{M}$ due to the many calculations that need to be done and therefore the need for Step 3 Dimensionality reduction.

Step 4: Increasing the training set's dimensionality. To each these calculations on the required individual faces, we calculate them from a reduced dimensionality covariance matrix.

Step 5: Choose K's best individual vectors so that $\mathrm{K}<\mathrm{M}$ can represent the entire training set. The yellow bars show the selected vectors of $\mathrm{K}$ Eigen which are adequate to represent the entire training set.

Step 6: Transform lower-dimensional K own vectors to the dimensionality of the original face.

Step 7: Each image is represented as a linear combination of all K own vectors plus the mean / average face.

Step 8: Calculate and store the corresponding weight vectors for each image in the training set.

\section{B. General Overview}

In this model, many similar components have been clustered to form sub-systems which then combine to form the whole system. Breaking the system into modules and sub-systems reveals the practical essence of the class attendance system. Fig. 1 flow diagram shows the operation of the processes.

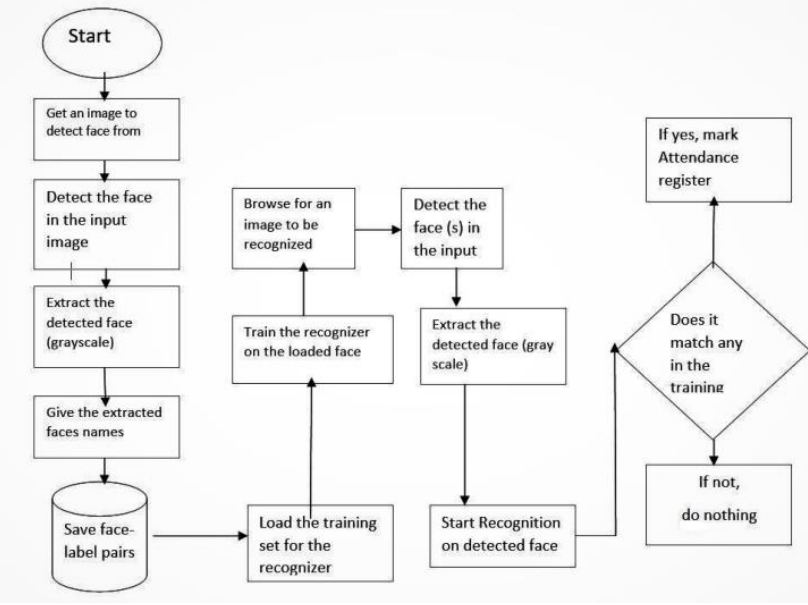

Fig. 1. Sequence of events in the class attendance system

Published By: 
From Fig. 1, we can see that most of the components used are identical only to be used at different stages of the face recognition process.

\section{Training Set Manager Sub System}

The training set management sub-system's conceptual architecture should consist of a component of image acquisition, a face detection feature, and a component of training set management. Together, to control the training collection, these elements work with the faces database. These will be developed in an application form for Windows.

\section{Face Recognizer Sub System}

The Face Recognizer's conceptual architecture would consist of the component of image processing, face recognizer, and face detection system all operating with the database of faces. Here, the portion of image acquisition and face detection is the same as those in the Training Set Manager sub-system as the same features. The only difference is the face recognizer component and its user interface controls. This will reload the training set on the added faces to train the recognizer and show the calculated Eigen-faces and average face. It should then display the recognized picture in a photo box

\section{E. System Architecture}

The system consists of a camera recording the student's photographs and submitting them to the server for image enhancement. The picture will appear in the Face Detection and Recognition modules after enhancement and then the presence will be identified on the server of the database. This is shown in Figure 2 of the experimental setup. Templates of individual student's face photos are stored in the Face database at the time of enrollment. The input image identifies all the faces here, and the algorithm compares them one by one with the face database. It saves a lot of time and this is a highly secure procedure that no one can mark someone else's attendance. Face database is the collection of face images and extracted features during the registration process and the second attendance inventory includes student information as well as uses to identify attendance. The figure below illustrates the three application subsystems ' conceptual design and implementation.

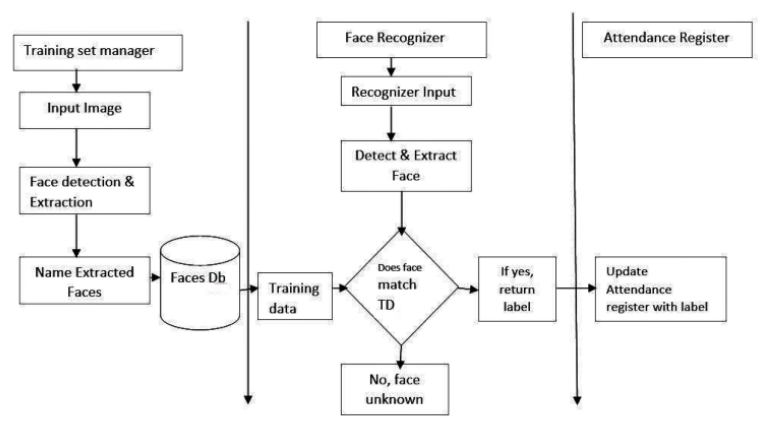

Fig. 2. The logical design of the Desktop Module Subsystems

\section{F. Functions of the two Sub-Systems}

The functionality of the components is shown in Figure 3 block diagrams. The face recognizer system consists of two main components, i.e. the manager of the training set and the face recognizer.

Those two modules must share the Faces database, the Face Detector system image acquisition; as they are similar in their features. Therefore, we must partition the machine into two subsystems and have to enforce their comprehensive conceptual designs.

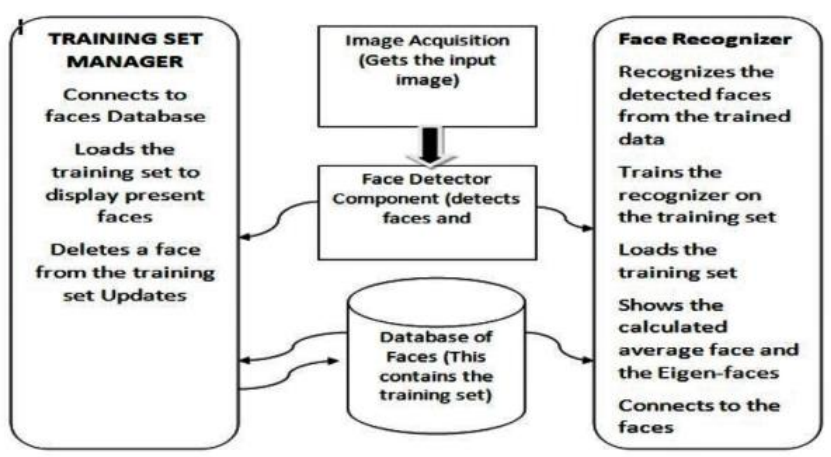

\section{Fig. 3. A block diagram showing functions of the components}

\section{G. Full Systems Logical Design}

The logical design of the full system are composed several components. The system first takes an image sample of student from input devices such as camera. Then we enhance the image using some image enhancement process for better face detection and recognition. After successfully identifying the face by comparing with face database the system can give attendance to the identified students and save it to attendance sheet.

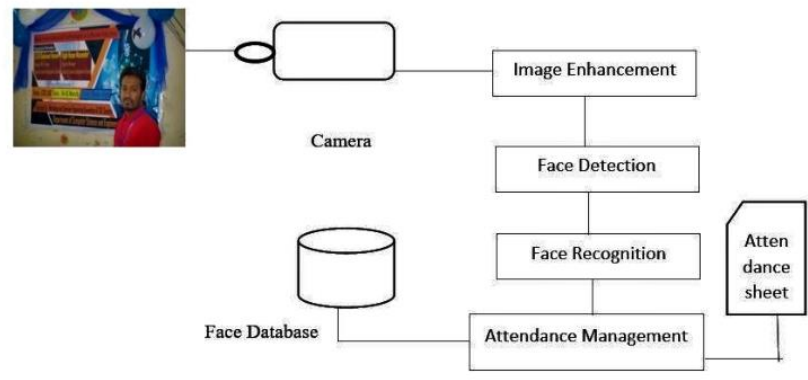

Fig. 4. Whole system logical design

\section{H. Tools Used}

We used the EmguCV Library to process images. EmguCV is a wrapper for the OpenCV image processing library. Visual Studio, Xamarin Studio and Unity can compile the wrapper, it can run on Windows, Linux, Mac OS X. EmguCV uses a form of face detector called a hair cascade. The Haar Cascade is a human face-trained classifier (detector). The attendance result sheet is stored on this paper folder in a text file. 


\section{Automatic Student Attendance System using Face Recognition}

\section{RESULT}

\section{A. User Interface of the System}

The attendance system adds faces to the training set for extracting feature. The image is taken from the marked red box as can be seen in Fig. 5 and displayed in the yellow box on a picture box. The Areas of Interest (AOI) i.e. face (es) in the drawing would be automatically identified by drawing a light yellow rectangular box. Then we assign a face mark to the blue rectal to the extracted gray scale.

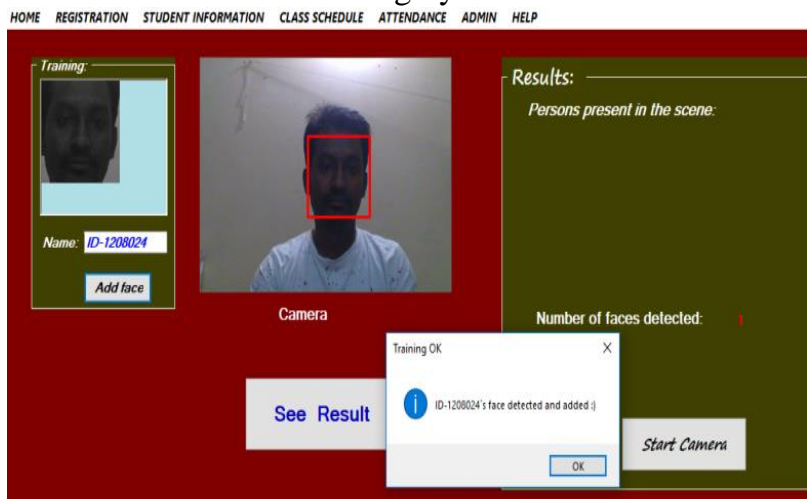

Fig. 5. Training set editor

Then we can adjust the pairs of face marks in the purple box if they are mistakenly identified or even eliminate the faces if they do not meet the criteria.

\section{B. The Face Recognizer}

The system match the observed image's input face to the faces captured during the recording process. If it is a match then it will retrieve the name associated with the input image.

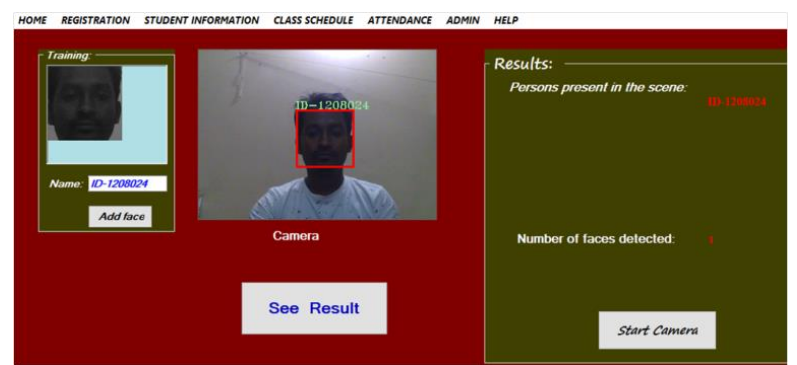

Fig. 6. Face recognizer with single student

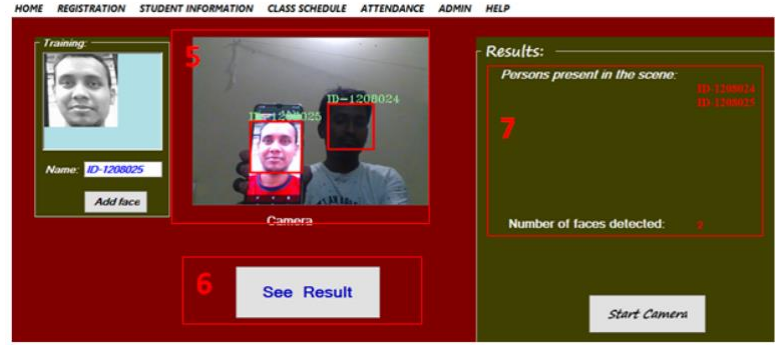

Fig. 7. Face recognizer with multiple students

Step 1 is for the marker to be qualified to recognize a face as known or unknown. Step 2 selects the recognizable face of the image source. This could be complemented by a live camera recorded images. The face input image will then be shown in the recognizer picture box 2 as shown in Figure 6. The input face ID of the image will then be shown as shown in Figure 7, Step 5.

The outlined box 1 shows the camera's present view / scene from Figure 8. The faces and eyes in the pictures are automatically marked as shown by the rectangular boxes around them. The face detected is extracted from and compared to the database. After a good match, the name associated with the face will be shown on the top edge of the rectangular box. Now shown in the highlighted box number 2 are the numbers of faces in the scene with their names. To connect faces to the database, use face press.

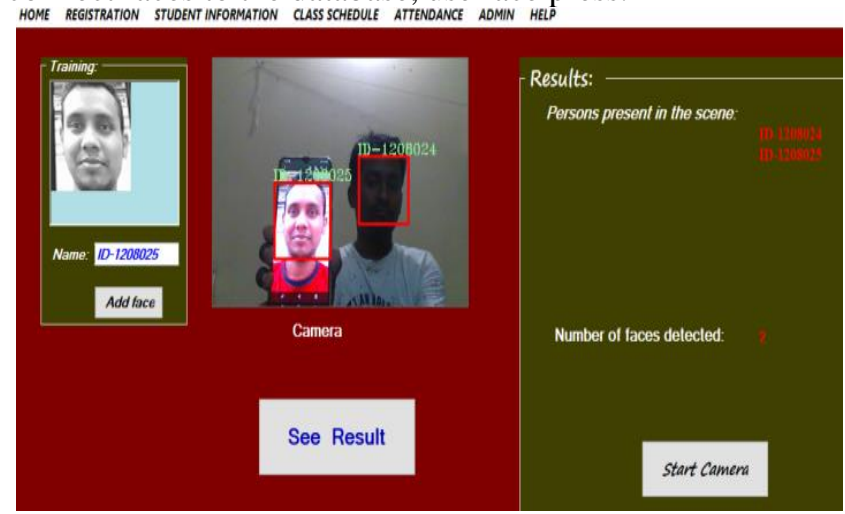

Fig. 8. Live camera feed window

To enhance the system's recognition performance, nine photos were selected for training for each person from the regular Trained Faces database; the remaining two photos were selected for the test collection. Of the fifteen subjects from the database of trained faces, twelve faces were recognized correctly. This was in proportion to the precision of $90 \%$. Time consuming faces where there is a large number of subjects involved.

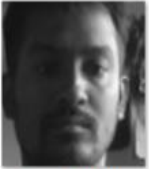

face1.bmp

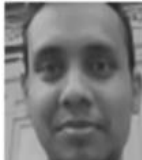

face2.bmp

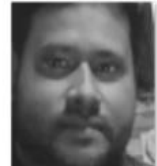

face3.bmp

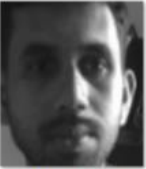

face4.bmp

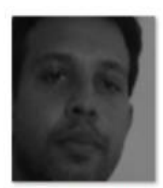

face5.bmp
Fig. 9. Images from class

\section{Attendance Sheet Creating and Marking}

When we click the CLICK HERE button a text file create in Face recognizer folder. In a new day create a new text file for attendance. In figure 10 shown the attendance register as a database.

$$
\begin{aligned}
& \text { 18-10-2019.txt - Notepad } \\
& \text { File Edit Format View Help } \\
& 1208024 \\
& 1208025 \\
& 1208017 \\
& 1208013 \\
& 1208010
\end{aligned}
$$

Fig. 10. Attendance register

The returned input face I d number was then used to fill the records in the database of the attendance registry. Clicking the Attendance sheet button will reveal the sheet as shown in figure 11 . The images are identified, monitored and recognized automatically in the video feed. Photos can also be transferred from the live to the database.

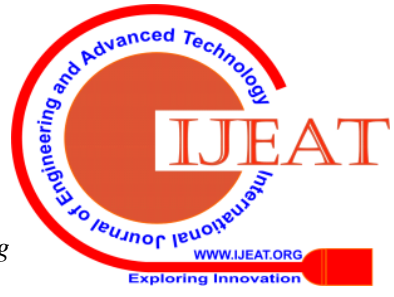




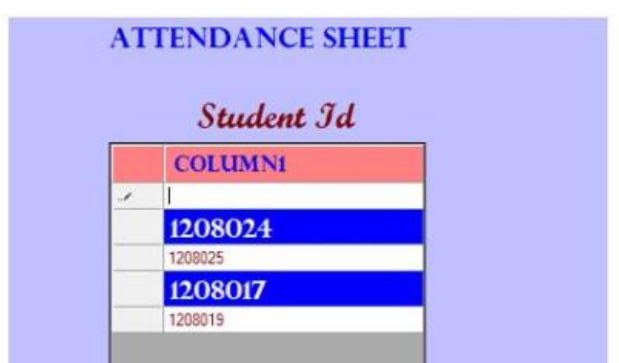

Fig. 11. The Attendance Sheet

\section{Analysis}

This work takes part in five stages:

Step 1: Training Data generation: The system that is the cropped images is initially trained and saved to the database and is detected and recognized. This data will also be used to compare the detected images in all the files uploaded and to mark the attendance.

Step 2: Capture: Use a tool to capture the video or image and upload the captured file to the server using the web application.

Step 3: Face Detection: Using the Haar-cascade classifier, the file uploaded to the server is detected face. The obtained frames are tested for the faces and for further identification they are cropped.

Step 4: Face Recognition: The images detected are correlated with the database's trained images. This now recognizes the detected images.

Step 5: Marking attendance: The recognized students are searched in the database after the recognition process and their attendance is marked.

Tests were carried out for several subsets specifically of the Yale dataset out of 60 faces in the sample. Tabulated the results obtained as in Table-I. The percentage identification rate for the various subsets was determined as the sum of the percentages. Recognition levels were not influenced by faces with or without glasses. The average percentage identification rate was $80.22 \%$. The middle light faces had the best overall ranking of $90 \%$. The main issues in the majority of the currently in use facial detection and recognition systems are rotation, orientation, distance of identification and lighting. Such issues reduce the system's performance unless it is carried out under the required constraints. Such restrictions would include putting the subjects in specific positions, which would be very challenging in a real world classroom environment and not to mention time consuming when there are a large number of subjects involved.

Table- I: Experimental results

\begin{tabular}{|c|c|c|c|c|}
\hline Datasets & $\begin{array}{c}\text { No of } \\
\text { Faces }\end{array}$ & $\begin{array}{c}\text { Successfully } \\
\text { detected } \\
\text { Faces }\end{array}$ & $\begin{array}{c}\text { Successfully } \\
\text { Recognized } \\
\text { Faces }\end{array}$ & $\begin{array}{c}\text { \% Correct } \\
\text { recognition }\end{array}$ \\
\hline $\begin{array}{c}\text { Center } \\
\text { light }\end{array}$ & 10 & 10 & 9 & 90 \\
\hline $\begin{array}{c}\text { Left } \\
\text { light }\end{array}$ & 15 & 15 & 11 & 73.3 \\
\hline $\begin{array}{c}\text { Right } \\
\text { light }\end{array}$ & 15 & 15 & 12 & 80 \\
\hline
\end{tabular}

\begin{tabular}{|c|c|c|c|c|}
\hline $\begin{array}{c}\text { Veiled } \\
\text { Faces }\end{array}$ & 10 & 10 & 7 & 70 \\
\hline $\begin{array}{c}\text { Bearded } \\
\text { Faces }\end{array}$ & 10 & 10 & 8 & 80 \\
\hline $\begin{array}{c}\text { Unveiled } \\
\text { A }\end{array}$ & 20 & 20 & 17 & 85 \\
\hline $\begin{array}{c}\text { Unveiled } \\
\text { B }\end{array}$ & 30 & 30 & 25 & 83.3 \\
\hline
\end{tabular}

With a different combination of methods and algorithms this system helps us to achieve the desired results more precisely. The minimum variable detection scale eliminates the identification and recognition distance issue for both close and group images. It improves the exactness of the face detection for upright faces to $100 \%$ and increases the precision of the face recognition by usually $70 \%$. Similarly, the setting of minimum neighbors enhanced the precise face detection significantly. The rectangular section of the observed face rather than the whole image captures and transform decreases the influence of background noise on the visual accuracy of the device.Histogram equalization is performed to the sample images, achieving uniform intensity distribution of the 46 output images through the reassignment of intensity pixels. All input images with different lighting are therefore enhanced in detail, leading to better facial recognition performance. Figure 12 shows the first 32 Eigen faces from a set of 50 faces created by each of the five men. The first few Eigen-faces display dominant facial features and the last 196 to 247 Eigen-faces, as shown in 13, are mostly picture noise and are therefore discarded. The average face from Figure 14 shows the smooth face structure of a generic human being.

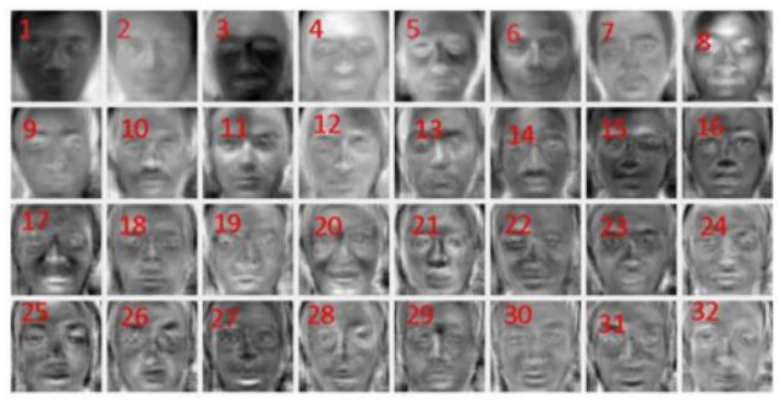

Fig. 12. The first 32 Eigen faces

The most prominent facial characteristics of the photos to be tested are found by the first Eigenfaces. The successor Eigen-faces (main components) shows the next highly likely facial features and more noise in consequence. Of the 247 training images, 195 main components and the average face are adequate to recreate the entire training kit entirely. We were therefore able to convert a set of correlated face variables (M) into a set of values of uncorrelated variables called own vectors. It was noted that the number of Eigen-faces is less than the original images of the face i.e. $\mathrm{K}$ $<$ M, PCA-based. 


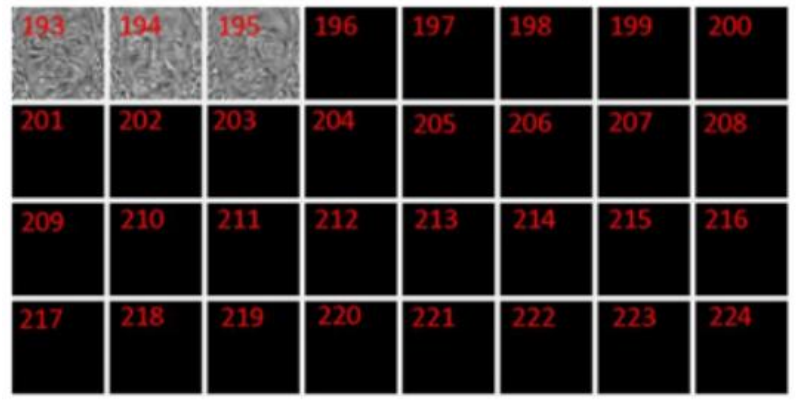

Fig. 13. The last Eigen-faces in the training set

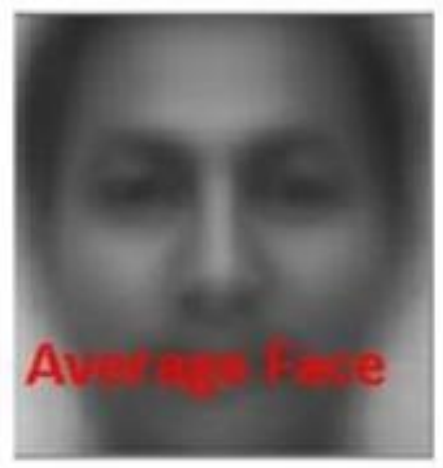

Fig. 14. The average face

Interestingly, the key component analysis can be used for compression of images from the Eigenfaces obtained in the face recognition stage, as shown by the dominant numbers of Eigen faces that can comfortably represent all images in the trainings package. Just 195 faces are required for the 247 pictures of the training set to completely re-create all the 247 faces.

\section{CONCLUSION}

A program has been suggested to ensure attendance. This replaces the manual system with a simple, reliable, cost-effective, time-saving automated system as it eliminates the stationary material and paper work. It is therefore predicted that this method would produce desired results and could be applied for logout in the future. In the near future, other strategies could also improve efficiency. We can say that a safe, stable, rapid and efficient class attendance management system was developed to replace a manual and inefficient system. The face recognition program saves time, reduces administration work and replaces obsolete electronic equipment for the currently in use stationery content. The device must not be mounted by specialized equipment, because it requires only a computer and a camera. The picture quality and performance must be tested in the real time scenario, particularly when the systems are operated from a live camera supply, and thus the camera plays a decisive role in the system's work. The mechanism can also be used for permission-based access control systems and for safe access authentication (restricted installations). The greatest threat to the system is spoofing. Anti-spotting techniques like eye twitch detection can be used in the event that the face recognition is provided by grabbed images for possible improvements to separate live from static images. A feature can therefore be added that lists all unidentified faces and can be manually checked by the user. Automatic attendance system has been developed to popularize errors. The efficient and reliable system of the attendance that can replace the old manual methods in the office environment. This method is sufficiently stable, accurate and ready for use. There is no need for specialized equipment in the office to mount the device. It can be designed with a camera and a computer. Authors intend to improve the effectiveness of face recognition by using the interaction between our system, users, and administrators in further work. On the other hand, our system can be used in a completely new face recognition application dimension, mobile face recognition, which can help ordinary people to know about any person being photographed by cell phone camera, including proper authorization to access a centralized database.

\section{REFERENCES}

1. Y. Kawaguchi and T. Shoji, "Face recognition-based lecture attendance system," Academic Center for Computing and Media Studie, 2005.

2. K. S. Kumar, S. Prasad, V. BhaskarSemwal, and R. C. Tripathi, "Real time face recognition using adaboost improved fast pca algorithm," Int. J. Artif. Intell.Appl, p. 45-58, 2011.

3. N. Kar and D. M. K. D. Barma, "Study of implementing automated at-tendance system using face recognition technique," International Journal of Computer and Communication Engineering, 2012.

4. T. Bradski and A. Kaehle, "Opencv ml algorithms," in Learning OpenCV. O'Reilly Media, Inc, 2008, p. 580.

5. J. Kanti and J. Papola, "Smart attendance using face recognition with percentage analyzer," International Journal of Advanced Research in Computer and Communication Engineering, vol. 3, 2014. [Online] Available:

https://ijarcce.com/wp-content/uploads/2012/03/IJARCCE10A-a-Jyot shana-smart-attendance.pdf.

6. J. Joseph and K. Zacharia, "Automated attendance management system using face recognition," International Journal of Science and research, vol. 2, 2013.

7. R. Tharanga, Samarakoon, Karunarathne, Liyanage, and D. Parer, "Smart Attendance using real time face recognition (smart-fr)," Semantic Scholar, 2013.

8. K. Selvi, P.Chitrakala, and A. Jenitha, "Face recognition based attendance marking system," IJCSMC, no. 3, p. 337 -342, 2014.

9. S. Chintalapati and M. Raghunad, "Automated attendance management system based on face recognition algorithms," International Conference on Computational Intelligence and Computing Research, 2013.

\section{AUTHORS PROFILE}

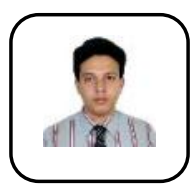

Partha Chakraborty is a member of the faculty at the Department of Computer Science and Engineering, Comilla University, Bangladesh is currently working as an assistant professor. He received a M.Sc. from the Jahangirnagar University, a renowned Bangladesh Public University, and a B.Sc. in the field of computer science and engineering. He also has an interest in computer vision, robotics, image processing and artificial intelligence for his work. To various international journals, he presented his research articles. He is actively involved in the fields of education and learing.

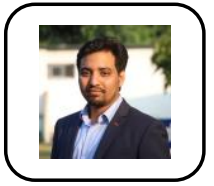

Chowdhury Shahriar Muzammel a faculty member of Comilla University, Bangladesh, Faculty of Engineering, is currently working as a lecturer in the Department of Computer Science and Engineering. He was awarded M.Sc. (Engineering) Bachelor's degree and B.Sc. (Technology) Comilla University degree in Computer Science and Engineering, a renowned Bangladesh public university. Signal processing, Bangla Natural Language Processing, Image Processing, and Artificial Neural Network are his current research interests. In various international journals, he presented his research articles. 
Mahmuda Khatun, a faculty member of the Faculty of Engineering, Comilla University, Bangladesh, is currently working as a Lecturer at the department of Computer Science and Engineering. She has completed her B.Sc. (Hons) in Computer Science and Engineering from Jahangirnagar University, Savar, Dhaka in 2016 and M.Sc. from the same university in 2018. Her teaching experience includes different graduate (M.Sc.) and under graduate courses. Her current research interest includes Wireless Communication, Machine Learning, Artificial Intelligence and Image Processing.

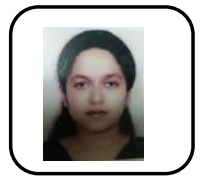

Sk. Fahmida Islam received the B.Sc. and M.Sc. degrees in computer science and engineering from Jahangirnagar University, Savar, Dhaka, Bangladesh.and currently a Ph.D student in the department of Computer Science and Engineering of Jahangirnagar University. Her current research interests include image processing, Internet of Things, vehicle tracking and mobile cloud computing.

Saifur Rahman is currently a student in the Department of Computer Science and Engineering of Comilla University, Bangladesh. His research interests are in Data Science, Machine Learning and Natural Language Processing. 